Musées, Patrimoine et Culture scientifiques et techniques

177 | 2018

mai-juin 2018

\title{
Fab Labs/Centres de science : même combat !
}

\section{Johan Langot}

URL : http://journals.openedition.org/ocim/2540

DOI : 10.4000/ocim.2540

ISSN : 2108-646X

\section{Éditeur}

OCIM

Édition imprimée

Date de publication : 1 mai 2018

Pagination : 18-21

ISSN : 0994-1908

Référence électronique

Johan Langot, «Fab Labs/Centres de science : même combat ! », La Lettre de l'OCIM [En ligne],

177 | 2018, mis en ligne le 01 mai 2019, consulté le 05 juin 2019. URL : http://journals.openedition.org/ ocim/2540; DOl : 10.4000/ocim.2540

Ce document a été généré automatiquement le 5 juin 2019.

Tous droits réservés 


\title{
Fab Labs/Centres de science : même combat!
}

\author{
Johan Langot
}

Le Propulseur, un Fab Lab nomade en Occitanie

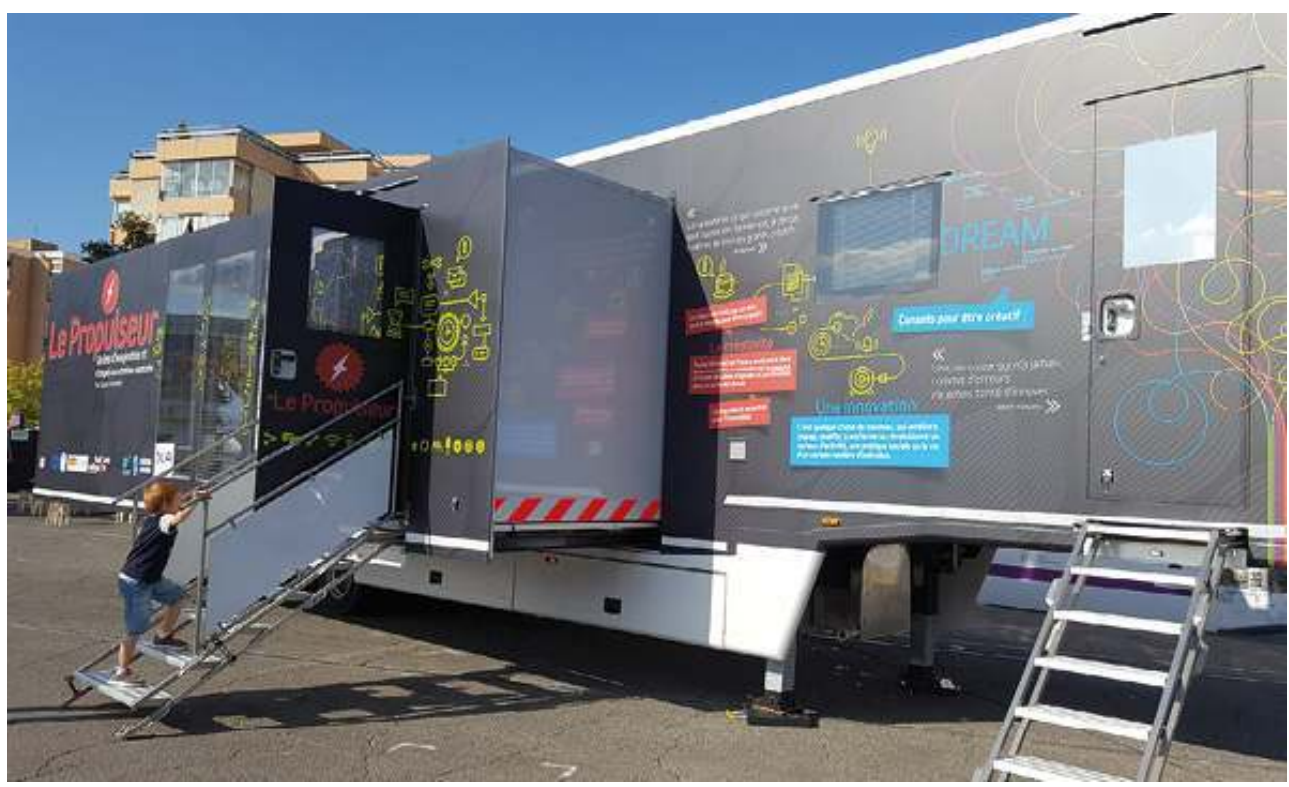

(c) Science Animation

\section{Élargir le champ des possibles}

En tant qu'acteurs de terrain, nous sommes persuadés que nous vivons un tournant dans la diffusion et l'appropriation par les publics des cultures scientifique, technique et industrielle (CSTI) comme celui qu'ont pu connaître les acteurs du développement durable il y a quelques années et qui a conduit à une appropriation par tous de cette problématique. Le moment est venu de sortir de notre "entre soi" de militants de la CSTI 
et de faire en sorte, qu'en cette période de remise en question de la science, un maximum d'acteurs et de structures, de tout horizon, s'emparent de nos thématiques et agissent pour un meilleur partage des savoirs, un développement de l'esprit critique et une réappropriation des technologies. Pour ce faire, en tant qu'acteurs "historiques", nous devons faire évoluer nos stratégies et démultiplier nos actions en nous appuyant sur d'autres secteurs que ceux avec qui nous travaillons traditionnellement (éducation, loisir...). En nouant notamment des partenariats avec des acteurs dans des domaines comme la lecture publique avec son réseau de bibliothèques implantées sur l'ensemble des territoires ; le secteur du tourisme qui touche un large public en saison; mais aussi avec des domaines émergents, depuis quelques années, comme ces nouveaux lieux, dits "tiers-lieux", tels que les Fab Labs ou Hackerspaces portés par le mouvement des Makers en pleine expansion. C'est ce que nous tentons de mettre en place en Occitanie dans le cadre du Pôle Territorial de Référence "Science et Société en Occitanie" en recensant tout d'abord ces acteurs, leurs besoins, et surtout en leur proposant des outils et des formations pour mettre en place des activités de CSTI dans leur lieu. 
Les Fab Labs, des lieux de diffusion et d'appropriation des savoirs et des savoir-faire

Le faire seul (Do-it-yourself) ou collectivement (Do-itwith-others), la démarche projet, la collaboration entre pairs, le partage... démontrent que ces valeurs portées par ces lieux en font de formidables outils de réappropriation des savoirs et des savoir-faire par les publics. L'intérêt est aussi de faire se croiser des publics très différents, scolaires, entrepreneurs, chercheurs ou encore artistes, qui vont partager des compétences. Les Fab Labs sont néanmoins généralement perçus comme des lieux hautement technologiques, attirant plus naturellement un public technophile avec un bagage technique important. L'enjeu est donc de démocratiser ces lieux et d'y attirer d'autres publics, plus jeunes, moins qualifiés, et plus féminins. Par ailleurs, même si ces lieux font actuellement l'objet d'une certaine "mode", qui risque de passer, c'est la démarche qui y est mise en œuvre qui est importante. Il s'agit donc de la faire perdurer en mobilisant tous les habitants de tous âges et de toutes origines sociales autour des enjeux, méthodes et pratiques de l'innovation sociale, culturelle, scientifique, technique et industrielle qui s'y développent.

\section{L'Occitanie, la première "Fab Région" de France}

2 En 2009, le premier Fab Lab de France, Artilect, a vu le jour à Toulouse. Et, naturellement, nos structures ont trouvé des points de convergence, pour, par exemple, alimenter nos expositions avec des projets développés dans le Fab Lab comme l'installation interactive présentée dans l'exposition Et si la plante idéale existait... Ou encore dans le cadre du programme Inmédiats qui a permis d'accompagner le développement des activités au démarrage du Fab Lab Artilect. Depuis, ces tiers-lieux atypiques ont essaimé dans la France entière, et particulièrement dans la Région Occitanie, première région de France à obtenir le label international "Fab Région". En effet, grâce à une volonté politique forte et un soutien financier, c'est plus de 50 Fab Labs qui ont ouvert ou sont en projet dans les 13 départements de la région. Avec d'autres, nous nous sommes rapidement impliqués dans la structuration de ce réseau au sein de la Fedlab : la Fédération des Fab Labs d'Occitanie. 
Implantés sur l'ensemble du territoire, et parfois dans des villages isolés, les Fab Labs deviennent de formidables relais pour la diffusion de la CSTI. Par ailleurs, nous nous impliquons fortement dans cette dynamique régionale avec le Propulseur, notre tiers-lieu - Fab Lab mobile, en tournée sur toute la région

\section{Le Propulseur, un lieu d'imagination et d'expérimentation nomade}

3 Le Propulseur s'inscrit dans une nouvelle génération de lieux, centrés sur l'implication des publics, la création des communautés et la culture de l'innovation. Plus qu'un simple outil, le Propulseur reflète à la fois l'essence de Science Animation par sa démarche d'itinérance et de rayonnement de la culture scientifique, technique et industrielle depuis plus de 30 ans, ainsi qu'un profond changement de modèle de participation des publics initié grâce au programme Inmédiats. En disposant d'un lieu d'accueil des publics (Science Animation ne dispose pas de lieu fixe) et d'outils numériques, Science Animation peut ainsi poursuivre son travail d'expérimentation de nouveaux formats de médiation scientifique et technique initié dans le cadre d'Inmédiats (nouvelles formes de narration, nouveaux formats d'ateliers participatifs, création de communautés...). Le Propulseur (www.science-animation.org/fr/le-propulseur-espace-itinerant-modulable) a été développé dans le cadre du programme Inmédiats et est financé par l'Europe à travers les fonds Feder, les Investissements d'Avenir, la Région Occitanie / Pyrénées-Méditerranée et Toulouse Métropole. Le Propulseur, espace mobile de $60 \mathrm{~m}^{2}$, propose de nombreux ateliers ludiques et créatifs ouverts à toutes et à tous : tests de technologies innovantes (imprimante 3D, découpe vinyle, découpe laser...), ateliers de fabrication et de créativité, animations scientifiques... Cet espace vise ainsi à créer l'émulation, stimuler la créativité et favoriser l'intelligence collective, en rendant plus accessibles, notamment aux jeunes, la technique et la culture de l'innovation. Installé sur une place de ville ou au cœur d'un quartier, ce tiers-lieu itinérant peut accueillir jeunes, parents, étudiants, artistes, entrepreneurs, passionnés et curieux pour co-construire des projets en lien avec la science, l'innovation et leur quotidien : créer un nouveau mobilier pour améliorer la ville, fabriquer une éolienne, imaginer les transports de demain... 
Une animation autour de l'imprimante 3D

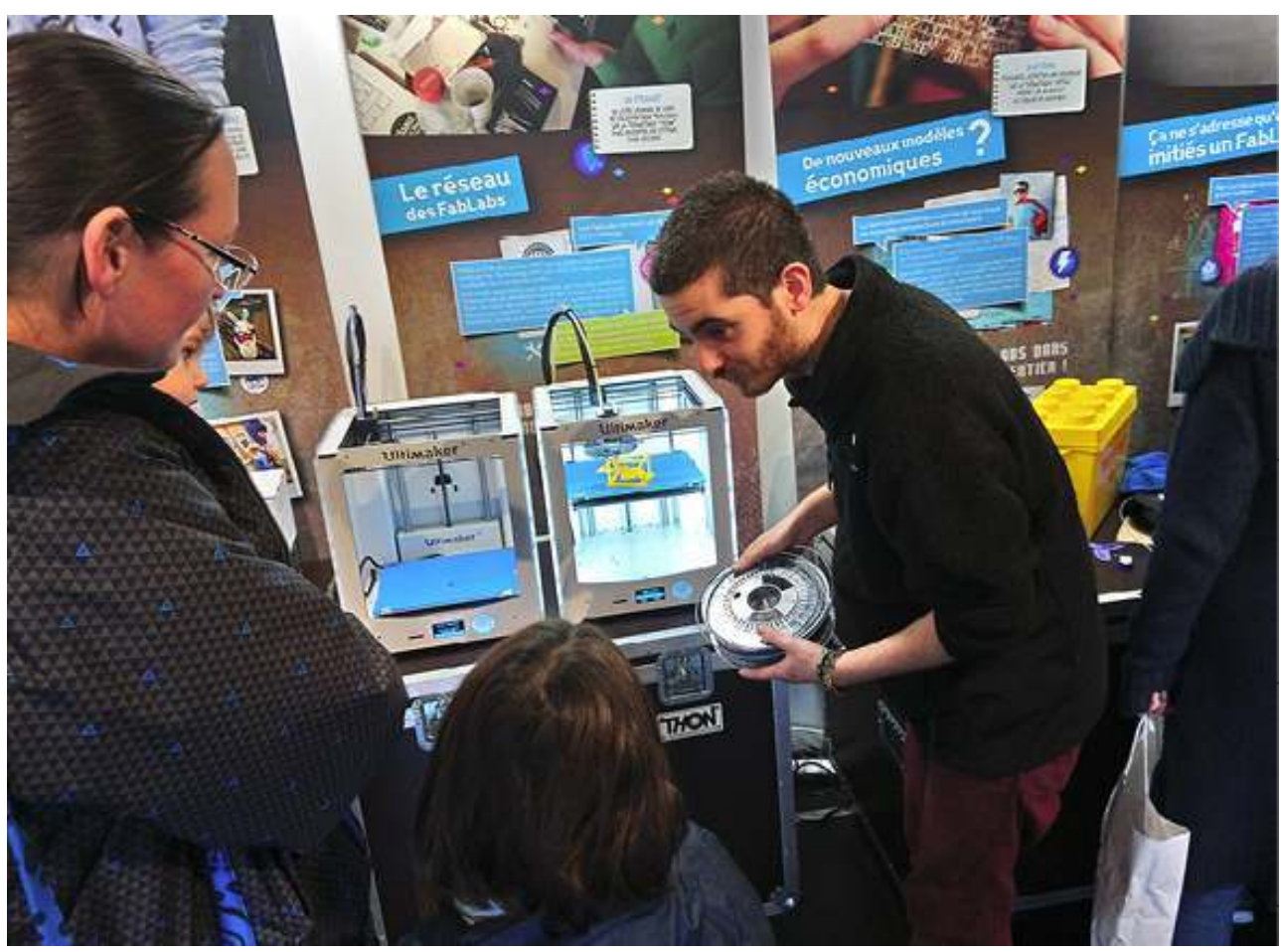

(c) Science Animation

4 Le Propulseur a débuté sa tournée en région en 2017 et la poursuit en 2018 pour accompagner la dynamique de développement des Fab Labs sur l'ensemble du territoire, et faire découvrir cet univers au grand public, et plus particulièrement aux jeunes : c'est ainsi plus de 30000 personnes qui ont participé aux animations du Propulseur. Il vient en appui des initiatives de développement de Fab Labs en sensibilisant les acteurs institutionnels et les entreprises à ces nouvelles démarches. Mais aussi et surtout, il a pour objectif d'inciter ces nouveaux lieux à intégrer l'ensemble des publics et en particulier les jeunes. Pour ce faire, les médiateurs à bord du Propulseur disposent de différents dispositifs, ateliers pratiques, démonstrations de machines, vidéos didactiques, expositions, formations... Depuis son lancement, le Propulseur a parcouru plus de 20 villes en région pour mener des projets très variés avec les acteurs locaux : 
L'assemblage d'une création réalisée à la découpeuse laser.

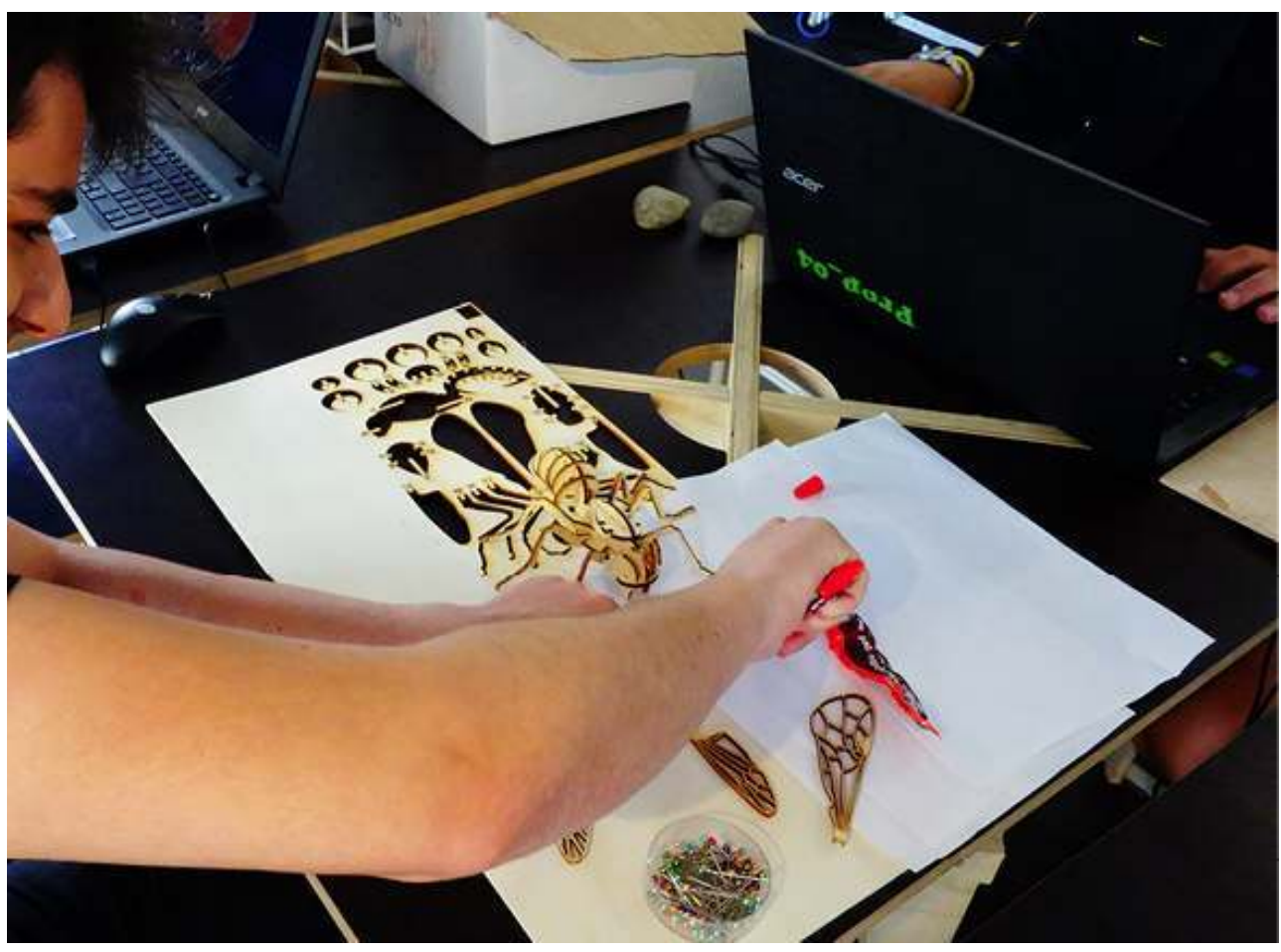

(C) Science Animation

plusieurs communes de la Métropole afin de sensibiliser et d'impliquer les citoyens autour du thème "Inventez la ville". Dans chaque ville, la programmation a été construite avec la collectivité autour de sujets tels que : améliorer les transports de la ville, favoriser le développement durable en ville ou encore rendre la ville plus ludique. Groupes scolaires et grand public ont participé à des ateliers de découverte autour de ces thématiques, des ateliers de créativité pour imaginer des solutions innovantes pour leur ville, ainsi que des ateliers de fabrication numérique pour concevoir les maquettes de leurs solutions.

- Projet Erasmus+ "SmartPupils"

Ce projet, en Ariège, consistait à accompagner une classe de collégiens afin de repenser leur collège. Ce projet a abouti à la conception par les élèves et les enseignants d'un jeu de société, dont le but est de construire le collège le plus innovant, prototypé au sein du Propulseur.

- Initiation aux machines numériques pour les scolaires

10 En déplacement pour une semaine d'animations et d'ateliers, le Propulseur a séjourné aux abords de la Maison du Savoir de Saint-Laurent-de-Neste dans les Hautes-Pyrénées pour accompagner le lancement du Fab Lab local. Plusieurs classes de tout niveau (CM, 3e, 3e SEGPA) ont pu profiter d'animations proposées autour de l'univers des Fab Labs et des machines telles qu'une imprimante 3D, une découpe laser et une découpe vinyle. Les élèves ont ainsi pu s'initier à ces outils avec l'aide de nos animateurs. 


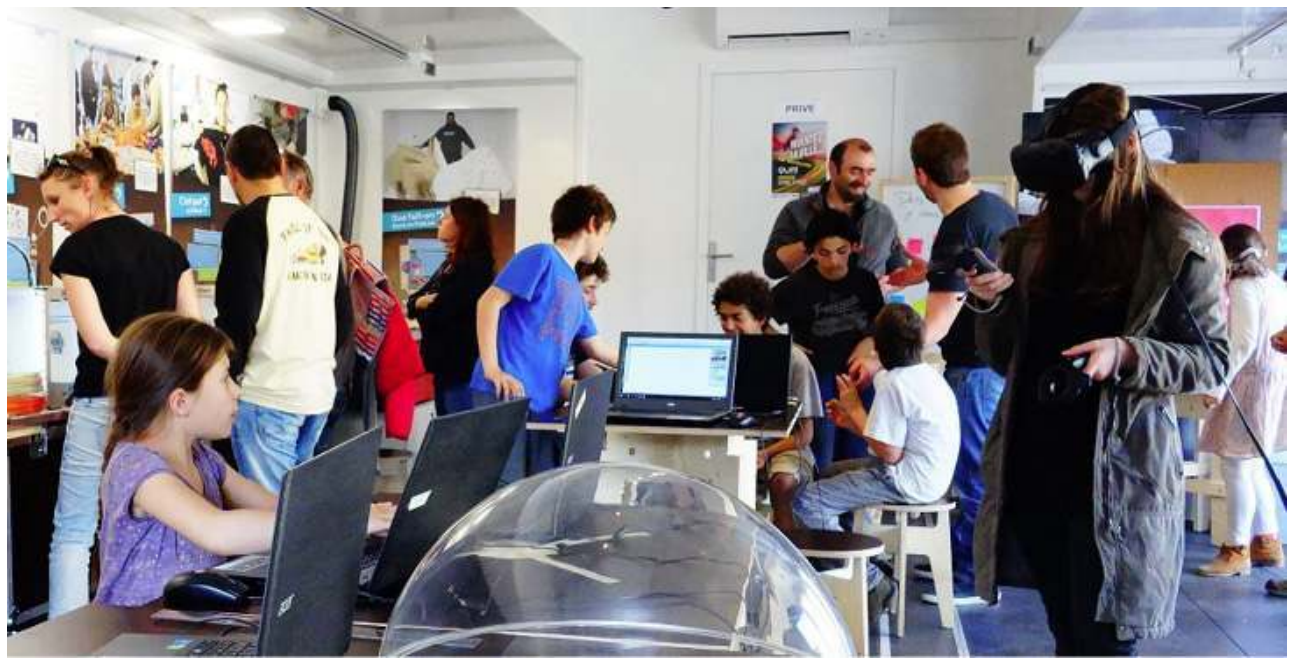

(c) Science Animation

\section{- Projet "Travailler pour nous" de l'Institut supérieur des arts de Toulouse}

Pendant deux semaines, un groupe d'étudiants a bénéficié du Propulseur pour mener, dans la ville de Caylus dans le Tarn et Garonne, un atelier de recherche autour du design et de l'habitation. Leur objectif était de penser des outils conviviaux destinés aux habitants, en effectuant des entretiens et en réalisant des séances de créativité avec ces derniers, puis en prototypant les outils à l'aide des machines à commande numérique et enfin, en leur proposant une démonstration du fruit de leur travail. Le partage de la culture scientifique, technique, industrielle et de l'innovation a toujours été un enjeu central pour la démocratie. À travers cette approche renouvelée, entreprenante et ancrée dans les territoires, l'appropriation par chacun d'une culture des sciences, des techniques et des innovations constitue aujourd'hui l'une des clés de l'avenir.

\section{Science Animation}

Ingénierie culturelle et médiation scientifique

Science Animation s'emploie à rendre accessibles à tous les sciences, les techniques et les innovations. Avec le concours de partenaires scientifiques, industriels et éducatifs, cette association conçoit des expositions, animations, rencontres ou dispositifs interactifs, pour favoriser l'intérêt, le plaisir, l'étonnement... L'envie de se plonger plus encore au cœur de la recherche et de l'innovation.

\section{Animation territoriale}

Science Animation anime un consortium d'associations partenaires afin de mailler l'ensemble de la région Occitanie et permettre à chaque individu d'avoir accès à la culture scientifique et technique. Ensemble, elles développent des ressources itinérantes, montent des projets d'envergure et animent l'ensemble du territoire. Elles participent aussi activement à l'animation du réseau d'acteurs, professionnels ou amateurs souhaitant participer au rayonnement de la culture scientifique et technique. 
Innovation culturelle

Science Animation s'appuie sur une équipe de permanents et de bénévoles investis et passionnés, issus des secteurs de la culture, des sciences, du numérique, de la communication, de l'événementiel, de la fabrication, du design... Depuis plusieurs années, ces équipes expérimentent de nouveaux formats de médiation scientifique et technique, en s'appuyant notamment sur les nouvelles technologies, la co-création, les démarches participatives ou encore la culture du divertissement. Pour mener à bien ses missions, Science Animation bénéficie du soutien du ministère de l'Enseignement supérieur, de la Recherche et de l'Innovation, de la Région Occitanie, de nombreuses collectivités territoriales (Conseils départementaux, Métropole de Toulouse et plusieurs municipalités), de nombreux organismes de recherche et universités, de partenaires privés, ainsi que de l'Europe pour certains projets à travers notamment les fonds Feder. Science Animation est, par ailleurs, membre et administrateur de l'Amcsti et membre du réseau européen Ecsite.

\section{RÉSUMÉS}

À partir de l'exemple du Propulseur, Fab Lab nomade, l'auteur montre comment, dans une perspective de démocratisation du partage de la culture scientifique, technique et industrielle, les Fab Labs sont aussi des lieux de diffusion et de co-construction des savoirs techniques à destination de tous les publics.

\section{INDEX}

Mots-clés : Fab Lab, réseau, CSTI

\section{AUTEUR}

\section{JOHAN LANGOT}

Directeur de Science Animation à Toulouse

johan.langot@science-animation.org 